\title{
Vitamin D status, bone mineral density and mental health in young Australian women: the Safe-D study
}

\author{
Emma T. Callegari, ${ }^{1}$ Nicola Reavley, ${ }^{2}$ Suzanne M. Garland, ${ }^{3-5}$ Alexandra Gorelik, ${ }^{6}$ \\ John D. Wark, ${ }^{1,7}$ on behalf of the Safe-D study team \\ ${ }^{1}$ Department of Medicine, Royal Melbourne Hospital, University of Melbourne, Parkville; \\ ${ }^{2}$ Melbourne School of Population and Global Health, University of Melbourne, Parkville; \\ ${ }^{3}$ Murdoch Children's Research Institute, Royal Children's Hospital, Parkville; ${ }^{4}$ Royal \\ Women's Hospital, Parkville; ${ }^{5}$ The University of Melbourne, Parkville; ${ }^{6}$ Melbourne \\ EpiCentre, Royal Melbourne Hospital, University of Melbourne, Parkville; ${ }^{7}$ Bone and \\ Mineral Medicine, Royal Melbourne Hospital, Parkville, VIC, Australia
}

\begin{abstract}
Significance for public health
Vitamin D deficiency, depression and osteoporosis are all major public health issues. Vitamin D deficiency has been associated with both reduced bone mineral density and depressive symptoms. Moreover, cohort studies have found that subjects with depression have lower bone mineral density when compared to healthy controls. Early adulthood is a critical time in young woman's lives as their independence, behaviours and lifestyle choices are established. These choices made as a young adult lay down the foundation for future health trajectories for not only for themselves but also for their potential partners and families. Addressing vitamin D deficiency, poor bone health and mental ill-health at a younger age may ultimately improve their wellbeing, productivity and long-term health outcomes. This study is of particular significance as the interplay between vitamin $\mathrm{D}$, depression and bone health is currently uncertain and such knowledge is crucial for understanding, prevention and treatment of these conditions.
\end{abstract}

\section{Abstract}

Background. Vitamin D deficiency has been associated with both poor bone health and mental ill-health. More recently, a number of studies have found individuals with depressive symptoms tend to have reduced bone mineral density. To explore the interrelationships between vitamin D status, bone mineral density and mental-ill health we are assessing a range of clinical, behavioural and lifestyle factors in young women (Part A of the Safe-D study).

Design and methods. Part A of the Safe-D study is a cross-sectional study aiming to recruit 468 young females aged 16-25 years living in Victoria, Australia, through Facebook advertising. Participants are required to complete an extensive, online questionnaire, wear an ultra-violet dosimeter for 14 consecutive days and attend a study site visit. Outcome measures include areal bone mineral measures at the lumbar spine, total hip and whole body, as well as soft tissue composition using dual energy x-ray absorptiometry. Trabecular and cortical volumetric bone density at the tibia is measured using peripheral quantitative computed tomography. Other tests include serum 25hydroxyvitamin D, serum biochemistry and a range of health markers. Details of mood disorder/s and depressive and anxiety symptoms are obtained by self-report. Cutaneous melanin density is measured by spectrophotometry.

Expected impact. The findings of this cross-sectional study will have implications for health promotion in young women and for clinical care of those with vitamin D deficiency and/or mental ill-health. Optimising both vitamin D status and mental health may protect against poor bone health and fractures in later life.

\section{Introduction}

Vitamin D deficiency, osteoporosis and depression are all major public health issues and contribute substantially to the global burden of disease. These conditions are most likely inter-related although the aetiology of the relationship is not currently well understood.

Vitamin D deficiency, defined by the Australian and New Zealand Bone and Mineral Society as serum 25-hydroxyvitamin D (250HD) levels of $<50 \mathrm{nmol} / \mathrm{L}$, is emerging as a global, public health issue in all age groups. ${ }^{1}$ In Australia, vitamin D deficiency affects 1 in 3 adults and is increasingly common in females, with more than $50 \%$ of Australian women having insufficient serum vitamin D in Winter-Spring.

Vitamin D is essential in achieving optimal musculoskeletal health. In children, long-term vitamin D deficiency can cause growth retardation and deformities in the skeleton. In adults, chronic vitamin D deficiency can lead to osteomalacia (defective bone mineralisation) and osteoporosis (a skeletal disease characterised by low bone mass and a subsequent increase in fracture risk). Each year there are an estimated 9.0 million fractures associated with osteoporosis globally resulting in significant mortality, disability and economic burden. The total health-care expenditure on musculoskeletal conditions in Australia in 2008-09 was $\$ 5.7$ billion. In 2012 , the national total direct and indirect costs associated with osteopenia and osteoporosis in Australia was AUD 2.75 billion: these costs will only increase with population ageing. Vitamin D deficiency is also associated with a number of poor health outcomes including an increase the risk of chronic health conditions such as diabetes, cardiovascular disease, some cancers, autoimmune diseases, infection and adverse mental health outcomes. Collectively, these health conditions affect millions of Australians, potentially leading to substantial reductions in vitality, economic loss and mortality.

Similar to vitamin D deficiency, mental disorders are also common worldwide. Worldwide prevalence estimates for mood disorders, such as depression, are between 3.3 and $21.4 \%$, while those for anxiety disorders are between 4.8 and $31.0 \%$. In Australia, almost half of the population aged 16-85 years (45\%) are estimated to have a mental disorder at some point in their lifetime. Females are more likely to experi- 
ence a 12-month mental disorder than males and the prevalence of 12month mental disorders is highest in young adults aged 16-24 years. Depression and anxiety disorders also account for most disabilityadjusted life years globally. Depression, in particular, contributes most of the non-fatal burden of mental and substance use disorders. It is characterised by sadness, irritability and anhedonia, in addition to a range of psychophysiological changes including sleep disturbance, weight change, feelings of worthlessness and suicidal ideation. Although the exact causes of depression are unknown it is likely to be the result of complex interactions between social, psychological and biological factors, one of which may be vitamin D deficiency.

The vitamin D receptor is found in numerous regions of the human brain, including the temporal, orbital and cingulate cortex, in the thalamus, in the accumbens nuclei, parts of the stria terminalis and amygdala and throughout the olfactory system. Vitamin D is suggested to have a number of functions within the human brain including the regulation of neurotrophic factors such as nerve growth factor, neuroprotection, neuroplasticity and modulating inflammation. The identification of vitamin D-responsive elements in the promoter region of serotonin receptors and tryptophan hydroxylase makes the link between mental disorders and vitamin D deficiency biologically plausible.

Animal models have shown that vitamin D can protect against methamphetamine-induced depletion of dopamine and serotonin, neurotransmitters that both play a role in depression. There are a number of cross-sectional studies suggesting a link between serum 250HD and depression. Anglin et al. compared 10 cross-sectional studies and demonstrated a strong association between vitamin D levels and increased risk of depression $(\mathrm{OR}=1.3,95 \% \mathrm{CI}$ : $1.0-1.7) .^{2}$ Similarly, a meta-analysis of cross-sectional and cohort studies found an inverse association between serum 250HD levels and the risk of depression. ${ }^{3}$ A recent prospective, nested, case-control study in 495 US military suicide cases found that individuals with lower 250HD levels had increased risk of suicide when compared to matched controls after adjustements for season of serum collection. ${ }^{4}$ Reviews of randomised controlled trials investigating the effects of vitamin D supplementation on depressive symptoms suggest that supplementation may only be beneficial in patients presenting with clinical depression but further, high-quality research with appropriate doses and outcome measures are needed to address the issue.

Although depression is not currently considered an established risk factor for osteoporosis, there is growing evidence that those with depression may be at greater risk of reduced bone mineral density (BMD). The strength of the association varies between studies, with one study finding patients with major depression had a 15\% deficit in lumbar spine BMD on average, when compared to healthy controls, ${ }^{5}$ while others have found no association between depression diagnosis or symptoms and BMD. ${ }^{6,7}$ In a meta-analysis of studies exploring the relationship between depression and reduced BMD, Cizza et al. ${ }^{8}$ found that BMD was reduced in subjects with depression at each skeletal site measured when compared to control subjects. In depressed patients, BMD was $4.7 \%$ lower at lumbar spine $(95 \% \mathrm{CI}-7.3 \%$ to $-2.2 \%, \mathrm{P}<0.001$; $\mathrm{n}=16$ studies), $7.3 \%$ lower at femoral neck $(95 \% \mathrm{CI}-10.7 \%$ to $-3.9 \%$; $\mathrm{P}<0.001 ; \mathrm{n}=8$ studies) and $3.5 \%$ lower at total hip $(95 \% \mathrm{CI}-5.7 \%$ to $-1.4 \%, \mathrm{P}<0.001 ; \mathrm{n}=13$ studies $).{ }^{8}$ It is most likely that a link exists between depression and osteoporosis mediated by factors such as cortisol, inflammatory markers and vitamin D. In patients who have sustained osteoporotic fractures it is possible that this link may be bidirectional in nature. Behavioural factors such as physical activity, smoking, alcohol and drug use may also mediate the relationship.

Taking into consideration the high prevalence of both depression and vitamin D deficiency in young women, it is clinically relevant to explore the possible links between these conditions and poor bone health together with lifestyle, behavioural and clinical factors that may mediate such associations. By looking at the strength and clustering of associations, this investigation may provide new insights into the possible causal relationships between these problems and thereby help efforts to reduce the risk of fracture in adult life. Hence, the aim of the study is to explore the interrelationships between vitamin D status, depression and osteopenia/osteoporosis in a cohort of young women.

\section{Design and methods}

The study protocol was approved by Melbourne Health Human Research and Ethics Committee, Melbourne Health, Victoria, Australia (project number 2013.007). The study is being carried out in accordance with the National Statement on Ethical Conduct in Research Involving Humans (2007) produced by the National Health and Medical Research Council of Australia. The Safe-D study is funded by National Health and Medical Research Council (NHMRC) project grant APP1049065.

\section{Study design}

The Safe-D study builds on the Young Female Health Initiative (YFHI) pilot study exploring health and well-being in young women. ${ }^{9}$ The Safe-D study comprises two parts: part A is a cross-sectional study of young women to explore the association between vitamin $D$ status and a range of important clinical health indices; part B is a randomised controlled trial to assess the effectiveness of an mHealth-based lifestyle intervention to improve vitamin D status and related health in young women with vitamin D levels ranging from $25-75 \mathrm{nmol} / \mathrm{L}$. This manuscript focuses on the study protocol for part A of the Safe-D study.

\section{Recruitment}

Young women aged 16-25 years currently living in Victoria, Australia, are recruited through a Facebook advertising campaign. ${ }^{9}$ Potential participants who click on the Safe-D study Facebook advertisement are redirected to a secure study website (www.safedstudy. org) where they are able to submit an expression of interest (EOI). Subjects who submit an EOI are contacted by telephone and given an overview of the study and their eligibility to participate is assessed. Young women under the age of 18 years are taken through a mature minor assessment by a study team member. ${ }^{9}$ Pregnant or breast feeding women are excluded from the study. Participants are recruited into the study if they are able to provide both verbal and written consent to complete all three study components; an online questionnaire, wear a sun monitoring device and attend a study site visit.

\section{Study package}

Each participant is sent a study package containing a Patient Information and Consent Form (PICF) used to obtain written consent. Included in the package is an ultra-violet (UV) dosimeter which participants are asked to wear on their wrist for 14 consecutive days. The UV dosimeters, used to measure personal UV exposure, were developed at the National Institute of Water and Atmospheric Research in New Zealand (manufactured by Scienterra, New Zealand) and have been set up and calibrated by the Australian Radiation Protection and Nuclear Safety Agency (ARPANSA, Melbourne, Australia). UV dosimeter data are downloaded and analysed to calculate average daily standard erythemal dose (SED) for each participant.

\section{Online questionnaire}

Participants are emailed links to an extensive questionnaire online which takes 1.5 to 2 hours to complete. The questionnaire is divided into 6 modules which participants can chose to complete in one sitting or one module at a time over the space of a week. Participants are 
asked questions regarding their medical history, including a diagnosis of depression or any other mental disorders, including age of diagnosis (if applicable). Participants may also report antidepressant use when asked about prescription medications used in the previous week. The Patient Health Questionnaire depression scale (PHQ-9) is used to assess depressive symptoms. It scores each of the nine Diagnostic and Statistical Manual of Mental Disorders, $4^{\text {th }}$ edition (DSM-IV) criteria as 0 (not at all) to 3 (nearly every day), to establish a depressive disorder diagnosis and grade its severity. The 7-item Generalised Anxiety Disorder Scale (GAD-7) is used to assess the probable cases of Generalised Anxiety Disorder (GAD) and measure symptom severity. Similar to the PHQ-9, it scales DSM criteria for GAD from 0 to 3 to give an overall score. Increasing scores are strongly associated with functional impairment and disability days. Other relevant information being collected in the questionnaire includes: race, age at menarche, hormonal contraceptive use, physical activity, smoking, alcohol use, sun exposure, calcium intake and vitamin D intake.

\section{Study visit}

Participants attend a 2-hour study site visit at the Royal Melbourne Hospital, Parkville, Victoria, Australia, that consists of:

\section{Bone densitometry}

Areal BMD is measured using dual energy X-ray absorptiometry (DXA) (QDR 4500A densitometer, Hologic Inc., Bedford, USA). Scans are analysed with QDR software version 9.1D. Areal BMD and bone mineral content (BMC) are measured at the lumbar spine (L1-L4), total hip and total body. Non-bone lean mass (kg), fat mass (kg) and percent body fat are also assessed. Peripheral quantitative computed tomography (pQCT) (XCT 3000, Stratec Medizintechnik GmbH, Pforzheim, Germany) is performed at the distal tibia (at the $4 \%$ site of the tibial length proximal to the distal end plate) and at the tibial shaft (at the $66 \%$ site) of the non-dominant leg. Scans are analysed with software version 5.50 to assess bone parameters including BMC, volumetric $\mathrm{BMD}$, total bone area (toA), cortical area (coA), cortical thickness (coTh), polar stress-strain index (SSIp) and bone strength index (BSI). Muscle cross-sectional area (MCSA) is also measured.

\section{Anthropometry}

A wall-mounted Holtain stadiometer (Holtain Ltd., Crymych, Dyed, UK) is used to measure height to the nearest $1 \mathrm{~mm}$. A mechanical beam balance scale (Continental Scale, Bridgeview, IL, USA) is used to measure body weight to the nearest $0.1 \mathrm{~kg}$. Body mass index (BMI) is calculated from these measurements (weight in kilograms divided by height in metres squared) and categorised into underweight, overweight or obese according to World Health Organisation (WHO) criteria.

\section{Biochemical assays}

Serum levels of $250 \mathrm{HD}\left(250 \mathrm{HD}_{3}\right.$ plus $\left.250 \mathrm{HD}_{2}\right)$ is measured using liquid chromatography tandem mass spectrometry (LC-MS/MS) at VivoPharm Laboratories (Melbourne, Australia). The $\mathrm{D}_{2}$ metabolite has a lower detection limit of $5.12 \mathrm{nM}$ while $\mathrm{D}_{3}$ is $6.94 \mathrm{nM}$. The intraassay coefficient of variation (CV) is $4.9 \%$ for total $250 \mathrm{HD}$ while the interassay CV for $250 \mathrm{HD}_{2}$ and $250 \mathrm{HD}_{3}$ is $3.7 \%$ and $1.6 \%$ respectively.

Bone turnover markers C-terminal telopeptide of type I collagen (CTX) and procollagen type 1 amino-terminal peptide (P1NP) as well as oestradiol are measured using the Roche Cobas e411 automated analyser (Roche Diagnostics GmbH, Mannheim, Germany). Serum biochemistry is measured using an Abbott ARCHITECT c16000 integrated system (Abbott Diagnostics, Abbott Park, IL, USA). Parathyroid hormone (PTH), testosterone, sex hormone bind globulin (SHBG) and prolactin are measured using an Abbott ARCHITECT i2000 SR immunoassay connected to a FlexLab track (Abbott Diagnostics).

\section{Spectrophotometry}

Cutaneous melanin density (i.e. skin colour) is measured using a 2500d Konica Minolta portable spectrophotometer (Osaka, Japan) coupled with a skin analysis program (CM-SA, Konica Minolta Sensing Inc., Osaka, Japan). Melanin density is assessed at the inner, upper arm (i.e. UV-unexposed region) and both the back of the hand and the cheek (i.e. UV-exposed regions). Three measurements are taken at each region. Spectrophotometer data are then exported from the software and the average of the triplicates is calculated to give a final melanin density for each location.

\section{Sample size and power}

It is necessary for the sample size for part A of the Safe-D project to provide sufficient eligible participants to recruit adequate numbers for part B of the study. Based on assumptions regarding increases in serum 250HD with vitamin D supplementation and taking into account study attrition rates, a recruitment target of 468 participants was set for part A. This sample size gives $80 \%$ power at $5 \%$ significance level to detect small-medium effect sizes (Cohen's $d=0.25-0.3$ ) in outcome measures, comparing participants with serum 250HD above and below the mean. This effect size corresponds to a $4 \%$ difference in lumbar spine and total hip BMD (standard deviation 13\%) and a 2.5\% difference in whole body BMD (standard deviation $9 \%$ ). ${ }^{10}$

\section{Discussion}

Adolescence and young adulthood are crucial life stages, during which the foundations for a woman's health across her lifespan are laid down. Ensuring optimal health during this time is extremely important in the prevention of a range of chronic health conditions, including obesity, diabetes, cancer and cardiovascular disease. To prevent musculoskeletal conditions such as osteoporosis and related fractures it is crucial to achieve optimal peak bone mass and offset bone loss in later life, whether this is by maintaining adequate vitamin D levels, improving calcium intake or perhaps the treatment of mental ill-health.

This study will provide insight into the prevalence of depressive symptoms and osteopenia/osteoporosis in young Australian women and may shed light on some of the lifestyle, behavioural and clinical characteristics that may put women at risk of poor bone health in adulthood. The aetiology of the relationship between bone mineral density and mental health, more specifically depression, and the direction of the link between these health domains is unclear at present but it likely to be multifactorial in nature (Figure 1). Few studies have examined

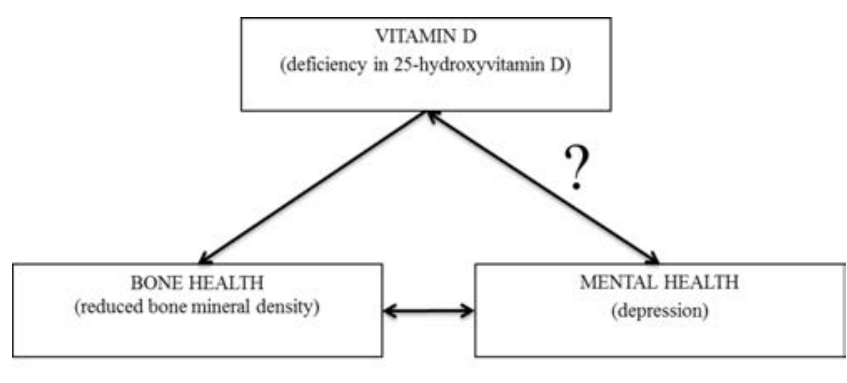

HOW MIGHT BONE HEALTH AFFECT MENTAL HEALTH?

Figure 1. The potential relationships between vitamin $D$, bone health and mental health. 
whether vitamin $\mathrm{D}$ is a common mediator between depression and reduced BMD. ${ }^{5,6,11-13}$ In those that have been tested for vitamin D, outcome measures that may affect an individual's 250HD levels such as skin colour, sun exposure, physical activity and diet were not fully examined. ${ }^{7,14}$ The Safe-D study is a comprehensive, multi-factorial study which is well-placed to enhance understanding of the links between mood, vitamin D and BMD in this currently under-represented demographic.

Previous studies investigating mental disorders and reduced BMD have suggested that mediating factors such as cortisol, serotonin or inflammatory cytokines (e.g. interleukin-6, interleukin-1, tumour

Correspondence: Emma T. Callegari, Department of Medicine, Level 4, Clinical Sciences Building, Royal Melbourne Hospital, Parkville, Victoria, Australia.

Tel.: +61.421.862.666 - Fax: +61.934.71863.

E-mail: emmatc@student.unimelb.edu.au

Key words: Vitamin D; bone mineral density; osteoporosis; depression; dual energy $\mathrm{x}$-ray absorptiometry.

Acknowledgements: the authors would like to thank the Safe-D chief investigators Associate Professor Marie Pirotta, Professor Anthony Jorm, Dr Shanton Chang and Professor George Varigos as well as associate investigators Professor Kim Bennell and Ms Adele Rivers. The authors would like to thank the Young Female Health Initiative associate investigators Dr Yasmin Jayasinghe, Dr Catherine Segan and Ms Stefanie Hartley. The authors would like to thank Marjan Tabesh, Anna Scobie and Skye Maclean for assisting with the Safe-D study and past YFHI co-ordinator Dr Elisa Young. We would like to acknowledge the following people for their help with various components of the study: Adrian Bickerstaffe (The University of Melbourne); Maria Bisignano (Melbourne Health Shared Pathology Service); Alison Brodie (Queensland University of Technology); Jessica Cargill (Australia National University); Dr Peter Farlie (Murdoch Childrens Research Institute); Dr Peter Gies (Australian Radiation Protection and Nuclear Safety Agency); Amanda Hawker (Murdoch Childrens Research Institute); Dr Ashwini Kale (The University of Melbourne); Dr Kerryn King (Australian Radiation Protection and Nuclear Safety Agency); Stefanie Koneski (Melbourne Health Shared Pathology Service); Professor Robyn Lucas (Australia National University); Jen Makin (Cancer Council Victoria); Professor Anne-Louise Ponsonby (Murdoch Childrens Research Institute); Rachel Slayter (Australia National University); Dr Ashwin Swaminathan (Australia National University); Oktay Tacar (Melbourne Health Shared Pathology Service); Dr Johannes Willnecker (Novotec Medical GmbH).

Contribution: ETC participated in the design of the study and drafted the manuscript. JDW, NR, SMG and AG are study investigators and were involved in study design, study co-ordination and helped draft the manuscript. AG is the study statistician and was involved in sample size and power calculations. All authors read, contributed to and approved the final manuscript. Conflicts of interest: The Safe-D study has received in-kind support from Swisse Wellness via the provision of vitamin D supplements for part B. Swisse Wellness did not play a role in study design nor the implementation of these studies.

Funding: The Safe-D study is funded by a National Health and Medical Research Council grant (APP1049065).

Received for publication: 6 July 2015.

Accepted for publication: 31 July 2015.

CCopyright E.T. Callegari et al., 2015

Licensee PAGEPress, Italy

Journal of Public Health Research 2015;4:594

doi:10.4081/jphr.2015.594

This work is licensed under a Creative Commons Attribution NonCommercial 3.0 License (CC BY-NC 3.0). necrosis factor alpha) may provide a link between these problems. ${ }^{15}$ The Safe-D study aims to also investigate a range of lifestyle factors which may affect the interrelationships between these health problems including physical activity, diet, smoking habits, alcohol and drug use. Improving health behaviours in young women will reduce the risk of chronic health problems in later life.

Current guidelines do not list depression as a risk factor for osteoporosis, although individuals taking selective serotonin reuptake inhibitors are listed as having a possible increased risk. Previous literature provides evidence that individuals with depression have greater reductions in BMD suggesting a mental health diagnosis such as depression should be taken into account when assessing an individual's risk of osteoporosis. By looking at the strength and clustering of associations between vitamin D deficiency, BMD and depression, the study may provide new insights into the determinants of vitamin D status and health outcomes in young women, as well as the possible causal relationships between these problems. If it is clear that a number of modifiable lifestyle and behaviour traits are associated with vitamin D deficiency, mental ill-health and/or poor bone health, these may be highlighted in national health promotion strategies. Public health messages may include encouraging safe sun exposure to improve vitamin D levels or promoting participation in physical activity. Promoting preventative behaviours and highlighting their importance to young women will put them in the best position to transition into adulthood at their healthiest. With vitamin D deficiency looming as a major public health issues it is crucial we gain a better understanding of vitamin D status, its determinants and its effect on biologically complex conditions such as osteoporosis and depression.

\section{References}

1. Mithal A, Wahl D, Bonjour JP, et al. Global vitamin D status and determinants of hypovitaminosis D. Osteoporosis Int 2009;20:180720.

2. Anglin RE, Samaan Z, Walter SD, McDonald SD. Vitamin D deficiency and depression in adults: systematic review and meta-analysis. Br J Psychiatry 2013;202:100-7.

3. Ju SY, Lee YJ, Jeong SN. Serum 25-hydroxyvitamin d levels and the risk of depression: a systematic review and meta-analysis. J Nutr Health Aging 2012:1-9.

4. Umhau JC, George DT, Heaney RP, et al. Low vitamin D status and suicide: a case-control study of active duty military service members. PloS One 2013;8:e51543.

5. Schweiger U, Deuschle M, Korner A, et al. Low lumbar bone mineral density in patients with major depression. Am J Psychiatry 1994;151:1691-3.

6. Amsterdam JD, Hooper MB. Bone density measurement in major depression. Prog Neuropsychopharmacol Biol Psychiatry 1998;22:267-77.

7. Yazıcı AE, Bagis S, Tot , et al. Bone mineral density in premenopausal women with major depression. Joint Bone Spine 2005;72:540-3.

8. Cizza G, Primma S, Coyle M, et al. Depression and osteoporosis: a research synthesis with meta-analysis. Horm Metab Res 2010;42:467.

9. Fenner Y, Garland SM, Moore EE, et al. Web-based recruiting for health research using a social networking site: an exploratory study. J Med Internet Res 2012;14:e20. 
10. Beasley JM, Ichikawa LE, Ange BA, et al. Is protein intake associated with bone mineral density in young women? Am J Clin Nutr 2010;91:1311-6.

11. Oikonen M, Hintsanen M, Laaksonen M, et al. Depressive symptoms are associated with lower bone mineral density in young adults with high job strain. The cardiovascular risk in young Finns study. Int J Behav Med 2014;21:464-9.

12. Williams LJ, Bjerkeset 0, Langhammer A, et al. The association between depressive and anxiety symptoms and bone mineral density in the general population: the HUNT study. J Affect Disord
2011;131:164-71.

13. Dorn LD, Susman EJ, Pabst S, et al. Association of depressive symptoms and anxiety with bone mass and density in ever-smoking and never-smoking adolescent girls. Arch Pediatr Adolesc Med 2008;162:1181-8.

14. Dorn LD, Beal SJ, Kalkwarf HJ, et al. Longitudinal impact of substance use and depressive symptoms on bone accrual among girls aged 11-19 years. J Adolesc Health 2013;52:393-9.

15. Michelson D, Stratakis C, Hill L, et al. Bone mineral density in women with depression. New Engl J Med 1996;335:1176-81. 